\title{
A Comparison of Remnants in Noncommutative Bardeen Black Holes
}

\author{
S. Hamid Mehdipour ${ }^{1, *}$ and M. H. Ahmadi ${ }^{1,+}$ \\ ${ }^{1}$ Department of Physics, College of Basic Sciences, Lahijan Branch, \\ Islamic Azad University, P. O. Box 1616, Lahijan, Iran
}

(Dated: September 9, 2021)

\begin{abstract}
We derive the mass term of the Bardeen metric in the presence of a noncommutative geometry induced minimal length. In this setup, the proposal of a stable black hole remnant as a candidate to store information is confirmed. We consider the possibility of having an extremal configuration with one degenerate event horizon and compare different sizes of black hole remnants. As a result, once the magnetic charge $g$ of the noncommutative Bardeen solution becomes larger, both the minimal nonzero mass $M_{0}$ and the minimal nonzero horizon radius $r_{0}$ get larger. This means, subsequently, under the condition of an adequate amount of $g$, the three parameters $g$, $M_{0}$, and $r_{0}$ are in a connection with each other linearly. According to our results, a noncommutative Bardeen black hole is colder than the noncommutative Schwarzschild black hole and its remnant is bigger, so the minimum required energy for the formation of such a black hole at particle colliders will be larger. We also find a closely similar result for the Hayward solution.

PACS numbers: 04.70.Dy, 04.50.Kd, 02.40.Gh, 04.20.Dw
\end{abstract}

Keywords: Regular Black Hole; Hawking Temperature; Noncommutative Geometry; Black Hole Remnant.

*Electronic address: mehdipour@liau.ac.ir

${ }^{\dagger}$ Electronic address: ahmadi@liau.ac.ir 


\section{INTRODUCTION}

The issue of central singularity of a Black Hole (BH) is an open problem in BH physics. Although it is commonly accepted that only a not yet accessible quantum theory of gravity would be competent to solve the problem appropriately, several phenomenological scenarios have been considered in the literature in order to study BHs with regular centers (for a review, see [1]). In 1968, Bardeen [2] introduced a compact object with an event horizon and without an intrinsic singularity, namely Bardeen $\mathrm{BH}$; it is the first regular $\mathrm{BH}$ model in general relativity. The Bardeen spacetime is spherically symmetric without violating the weak energy condition and the inside of the horizon is deSitter-like wherein the matter has a high pressure. In 2000, Ayón-Beato and García [3] reinterpreted the Bardeen model as the gravitational field of a nonlinear magnetic monopole. A few years later, Hayward [4] investigated the formation and evaporation of a new kind of the regular solution, i.e. Hayward BH, in which its static region is Bardeen-like and the dynamic regions are Vaidyalike. Recently, a family of rotating regular solutions have been obtained by applying the Newman-Janis algorithm to the Hayward and to the Bardeen spacetimes [5]. Afterwards, a general class of regular solutions using a general mass term described by a function which generalizes the Bardeen and Hayward mass terms have been constructed [6]. The regular BHs have extensively been studied in the recent literature (see for instance, [7- 15]).

Besides, the authors of Refs. [16 20] have utilized a spherically symmetric matter distribution leading to no curvature singularity. In fact, they have presented a physically inspired type of noncommutativity corrections to $\mathrm{BH}$ solutions. In this method, the point-like structure of mass, instead of being totally localized at a point, is described by a smeared structure throughout a region of linear size $\sqrt{\theta}$. In other words, the mass density of a static, spherically symmetric, particle-like gravitational source cannot be a delta function distribution, but will be given by a Gaussian distribution $\rho_{\theta}(r)=M /(4 \pi \theta)^{3 / 2} \exp \left(-r^{2} / 4 \theta\right)$. It has been demonstrated that the modified metric does not allow the $\mathrm{BH}$ to decay below the Planckian relic. The evaporation process ends when the size of the $\mathrm{BH}$ reaches a Planck-sized remnant, explaining the $\mathrm{BH}$ released from the curvature singularity at the origin. Here, the regularity of the metrics emerges from the appearance of a minimal length preparing a natural cut-off at small scales. The idea of a minimal length is confirmed by many outcomes of various approaches to quantum gravity [21 25]. This universal cut-off is entered into the energy- 
momentum tensor of the Einstein equations, and stands for the degree of delocalization of the matter distribution [26-28].

From the other viewpoint, all the various arguments concerning the so-called Hawking information loss paradox rely on semi-classical methods and guess about the behavior of systems in the quantum gravitational regime, but there are substantial struggles over the success of the arguments. The basis of the information loss problem turns back to Hawking's discovery that the theory of quantum fields in a curved spacetime indicates that BHs will emit thermal radiation at a temperature inversely proportional to their mass [29]. Conservation of energy points out that the $\mathrm{BH}$ will lose mass through this procedure, and if nothing stops the evaporation of the $\mathrm{BH}$ will ultimately cease to exist. This proposition of total evaporation is necessary for Hawking's argument, and may be refused by a remnant proposal that we will be considering in this paper (for reviews on resolving the paradox, see [30 36]). In other words, Planck scale physics may terminate the Hawking radiation and prohibit the appearance of a singularity in the center of a $\mathrm{BH}$, i.e. the appearance of a $\mathrm{BH}$ remnant; for

example, in a recent result by Paul and Majhi [37], the nature of the cascade of Hawking emission spectrum in the presence of a back reaction was studied. They observed that under a physical background, below a particular value of the mass, which is of the Plank mass order, the Hawking radiation must stop wherein a remnant is formed.

In this work we shall include the influences of inspired noncommutativity to the one of the most popular models of non-singular BHs, i.e. the Bardeen BH, and analyze the remnants of Noncommutative Bardeen (NB) BHs. Afterwards, we consider the thermodynamic properties of the NB solution, providing its Hawking temperature. Throughout this paper natural units are used with the following definitions: $\hbar=c=G=k=1$.

\section{THE NB SOLUTION}

In this section we first include the noncommutative effects in the line element of Bardeen and then analyze the consequences of the resulting metric. Now, let us start from the Schwarzschild-like class of metrics which describe the spacetimes in the so-called Kerr-Schild form and in the presence of matter

$$
d s^{2}=d s_{M}^{2}-\frac{f(r)}{r^{2}}\left(k_{\mu} d x^{\mu}\right)^{2}
$$


where $d s_{M}^{2}$ is the Minkowski line element expressed in a spherical basis and $k_{\mu}$ is a null vector in Minkowski coordinates. The expression $f(r)$ is found to be

$$
f(r)=2 m(r) r
$$

According to the Kerr-Schild decomposition, the above equation has a general validity, so its generic structure is kept and it is not sensitive to different forms of the mass term $m(r)$. For the Bardeen metric we have

$$
m(r)=M\left(\frac{r^{2}}{r^{2}+g^{2}}\right)^{\frac{3}{2}}
$$

where $g$ is the magnetic charge of the BH. Now, using the noncommutativity approach [16 20], the metric describing the noncommutative geometry inspired Bardeen $\mathrm{BH}$ is given by

$$
d s^{2}=\left(1-\frac{2 m(r)}{r}\right) d t^{2}-\left(1-\frac{2 m(r)}{r}\right)^{-1} d r^{2}-r^{2} d \Omega^{2},
$$

where $m(r)$ can now be written in terms of the smeared mass distribution $M_{\theta}$ as follows:

$$
m(r)=M_{\theta}\left(\frac{r^{2}}{r^{2}+g^{2}}\right)^{\frac{3}{2}}
$$

and $M_{\theta}$ can implicity be given in terms of the lower incomplete Gamma function,

$$
M_{\theta}=\int_{0}^{r} \rho_{\theta}(r) 4 \pi r^{2} d r=\frac{2 M}{\sqrt{\pi}} \gamma\left(\frac{3}{2} ; \frac{r^{2}}{4 \theta}\right) .
$$

The radiating behavior of such a noncommutative regular $\mathrm{BH}$ can now be easily investigated by plotting the temporal component of the metric, $g_{00}$, versus the radius $r$ for an extremal BH with different values of $g$ (see Fig. (1)). The plot presented in Fig. 1 shows, for several values of minimal nonzero mass $M_{0}$, the possibility of having an extremal configuration with one degenerate event horizon as the parameter $g$ grows. As this figure shows, the coordinate noncommutativity leads to the existence of a remnant mass in which the NBBH can shrink to.

The line element (44) has a coordinate singularity at the event horizon radius, $r_{H}$, that can be obtained from the equation $g_{00}\left(r_{H}\right)=0$ as follows

$$
1-\frac{2 m\left(r_{H}\right)}{r_{H}}=0
$$

with

$$
m\left(r_{H}\right)=M_{\theta}\left(r_{H}\right)\left(\frac{r_{H}^{2}}{r_{H}^{2}+g^{2}}\right)^{\frac{3}{2}}
$$




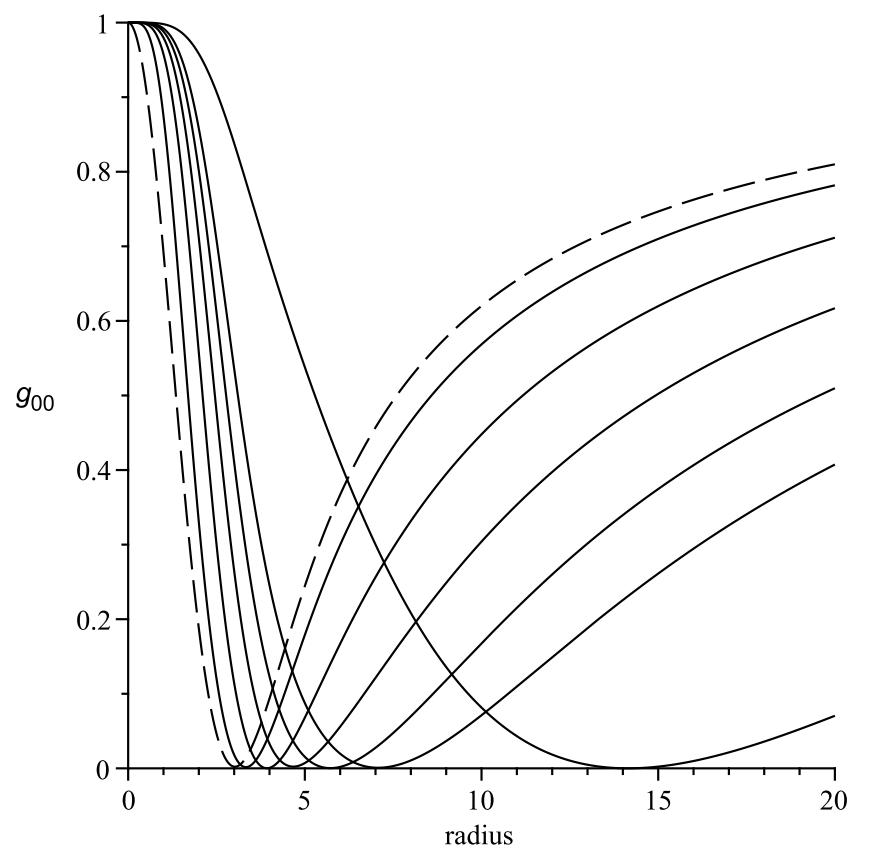

FIG. 1: The temporal component of the metric, $g_{00}$, versus the radius $r / \sqrt{\theta}$ for different values of $g / \sqrt{\theta}$. The figure shows the possibility of having an extremal configuration with one degenerate event horizon at $M=M_{0}$ (extremal NBBH). This shows the existence of a minimal nonzero mass $\left(M_{0}\right)$ that the $\mathrm{BH}$ can shrink to. On the right-hand side of the figure, from top to bottom, the solid lines correspond to the NBBH for $g=1.00 \sqrt{\theta}, 2.00 \sqrt{\theta}, 3.00 \sqrt{\theta}, 4.00 \sqrt{\theta}, 5.00 \sqrt{\theta}$, and $g=10.00 \sqrt{\theta}$, respectively. The dashed line refers to the Schwarzschild case so that it corresponds to $g=0$.

The analytical solution of Eq. (17) for $r_{H}$ in a closed form is impossible, but it is possible to solve it to find $M$, which provides the mass of the NBBH as a function of the horizon radius $r_{H}$. This leads to

$$
M=\frac{r_{H}}{2\left(\frac{r_{H}^{2}}{r_{H}^{2}+g^{2}}\right)^{\frac{3}{2}}\left[\mathcal{E}\left(\frac{r_{H}}{2 \sqrt{\theta}}\right)-\frac{r_{H}}{\sqrt{\pi \theta}} e^{-\frac{r_{H}^{2}}{4 \theta}}\right]},
$$

where the Gauss error function $\mathcal{E}(x)$ is defined by $\mathcal{E}(x) \equiv 2 / \sqrt{\pi} \int_{0}^{x} e^{-p^{2}} d p$. The results of the numerical solution of the mass as a function of the horizon radius are displayed in Fig. 2, As expected, from the mass equation (9), the noncommutativity indicates a minimal nonzero mass in order to have an event horizon. So, in the noncommutative case, for $M<M_{0}$ there is no event horizon.

For more details, the numerical results for the remnant size of the $\mathrm{BH}$ and for different

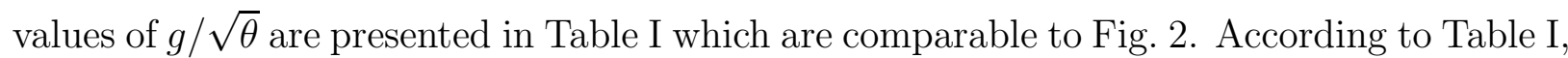
as $g$ increases both the minimal nonzero mass and the minimal nonzero horizon radius are enlarged which subsequently lead us to the final result:

$$
g \propto M_{0} \propto r_{0} .
$$




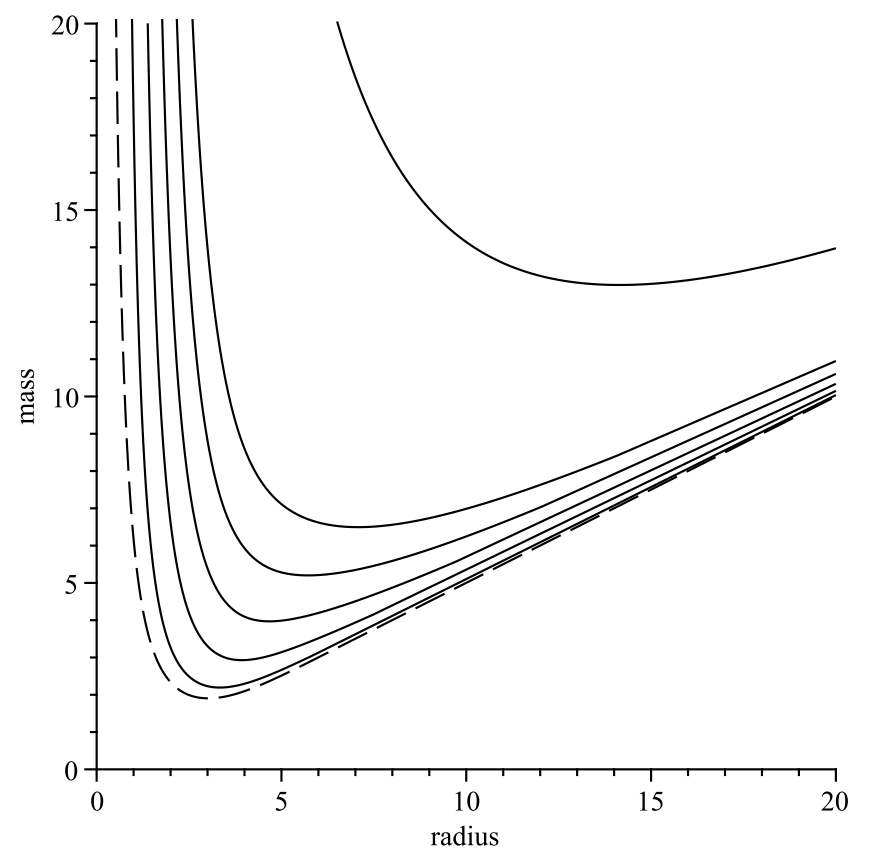

FIG. 2: The mass of the NBBH, $M / \sqrt{\theta}$, versus the event horizon radius, $r_{H} / \sqrt{\theta}$, for different values of $g / \sqrt{\theta}$. On the right-hand side of the figure, from bottom to top, the solid lines correspond to the NBBH for $g=$ $1.00 \sqrt{\theta}, 2.00 \sqrt{\theta}, 3.00 \sqrt{\theta}, 4.00 \sqrt{\theta}, 5.00 \sqrt{\theta}$, and $g=10.00 \sqrt{\theta}$, respectively. The dashed line refers to the Schwarzschild case so that it corresponds to $g=0$.

This means in the limit $g / \sqrt{\sigma} \gg 1$, the magnetic charge is proportional to the remnant mass and to the remnant radius.

Here, it should be emphasized that the physical interpretation of the noncommutative parameter $\theta$ is the smallest fundamental cell of an observable area in noncommutative geometry, in the same way that the Planck constant $\hbar$ explains the smallest fundamental cell of an observable phase space in quantum mechanics. The scale of $\sqrt{\theta}$ is, possibly and most reasonably, of the order of an inverse characteristic energy of the Planck scale. Most of the phenomenological examinations of the noncommutativity models have assumed that the noncommutative energy scale cannot lie far above the TeV regime [38-41]. Given that the fundamental Planck scale in models with large extra dimensions is as small as a TeV, for solving the hierarchy problem [42-45], therefore it could be possible to set the noncommutative effects in a TeV energy scale.

In this process, the minimum value of the NBBH mass increases to a value more than its value for the noncommutative Schwarzschild one (see Table【). Thus, in the theory of regular BHs, if the parameter $g$ becomes sufficiently large, i.e. $g / \sqrt{\theta} \gg 1$ with a sufficiently small noncommutative inverse length parameter $(1 / \sqrt{\theta} \sim 1 \mathrm{TeV})$, then the noncommutativity effect can concretely decrease the possible formation and detection of $\mathrm{BHs}$ in $\mathrm{TeV}$-scale 
TABLE I: The minimal nonzero mass of the NBBH (remnant mass, $M_{0} / \sqrt{\theta}$ ) and also the minimal nonzero horizon radius, $r_{0} / \sqrt{\theta}$, for different values of $g / \sqrt{\theta}$. For a large amount of $g / \sqrt{\theta}$, i.e. $g / \sqrt{\theta} \gg 1$, there is a linear relationship between the remnant mass and the remnant radius. As can be seen from the table, the results are comparable to Fig. 2

\begin{tabular}{c|c|c}
\hline \hline \multicolumn{3}{c}{ NBBH } \\
\hline Magnetic Charge & Remnant Mass & Remnant Radius \\
\hline$g=0$ & $M_{0} \approx 1.90 \sqrt{\theta}$ & $r_{0} \approx 3.02 \sqrt{\theta}$ \\
\hline$g=1.00 \sqrt{\theta}$ & $M_{0} \approx 2.19 \sqrt{\theta}$ & $r_{0} \approx 3.33 \sqrt{\theta}$ \\
\hline$g=2.00 \sqrt{\theta}$ & $M_{0} \approx 2.92 \sqrt{\theta}$ & $r_{0} \approx 3.92 \sqrt{\theta}$ \\
\hline$g=3.00 \sqrt{\theta}$ & $M_{0} \approx 3.96 \sqrt{\theta}$ & $r_{0} \approx 4.67 \sqrt{\theta}$ \\
\hline$g=4.00 \sqrt{\theta}$ & $M_{0} \approx 5.20 \sqrt{\theta}$ & $r_{0} \approx 5.72 \sqrt{\theta}$ \\
\hline$g=5.00 \sqrt{\theta}$ & $M_{0} \approx 6.49 \sqrt{\theta}$ & $r_{0} \approx 7.07 \sqrt{\theta}$ \\
\hline$g=10.00 \sqrt{\theta}$ & $M_{0} \approx 12.99 \sqrt{\theta}$ & $r_{0} \approx 14.14 \sqrt{\theta}$ \\
\hline$g=100.00 \sqrt{\theta}$ & $M_{0} \approx 129.90 \sqrt{\theta}$ & $r_{0} \approx 141.42 \sqrt{\theta}$ \\
$\vdots$ & $\vdots$ & $\vdots$ \\
\hline$g \gg \sqrt{\theta}$ & $\Longrightarrow \quad g \propto M_{0} \propto r_{0}$ \\
\hline \hline
\end{tabular}

collisions at particle colliders, such as the Large Hadron Collider (LHC) [67] and the UltraHigh Energy Cosmic Ray (UHECR). However, if the fundamental Planck scale is of the order of a few $\mathrm{TeV}$, then the LHC may produce BHs. These BHs may have masses on the order of $\mathrm{TeV}$. In this sense, on the other hand, the complete decay of BHs is impossible and the final Planck-sized remnant can be thought of as the order of $\mathrm{TeV}$.

Based on our computations, the total evaporation of the $\mathrm{BH}$ is not possible in principle. Therefore, the idea of a stable BH remnant as a candidate to conserve information has fixed. Note that, currently there are some proposals about what happens to the information that falls into a BH. One of the main proposals is that the $\mathrm{BH}$ never disappears completely, and the information is not lost, but would be stored in a stable remnant. A remnant proposal reflects the fact that the semi-classical approaches used to derive the Hawking effect are obviously inapplicable when the $\mathrm{BH}$ reaches the Planck mass. Possibly, Planckian physics will propose some way of conserving the information held in the BH.

The remnant proposals are generally divided into two categories. The first is stable 
remnants and the second is long-lived remnants. If a remnant is stable, then Planck scale quantum gravitational effects shut down the Hawking radiation, and the BH remnant continues to exist for all future time. Inasmuch as the BH, and its information, are prohibited from vanishing totally, the troubling result of Hawking's argument, i.e. the non-unitary evolution is prevented. For example, in Ref. [46], we have shown that, as a well-known result of the spacetime noncommutativity, a part of information may be preserved in a stable $\mathrm{BH}$ remnant. On the other hand, the process for long-lived remnants is completely different. They eventually disappear. In spite of the fact that the semi-classical models imply that no information can escape in the Hawking radiation, these models will fail in Planckian processes. This scenario is inspired by this cognition that the physics at the Planck scale might return the information to the external universe, and once the coherence of the external universe is made safe, the remnant could safely disappear. As an example of long-lived kinds of remnants, one can point to Ref. [47] in which an exact $(t-r)$ dependent case of a noncommutative Schwarzschild-like metric for a Vaidya solution was calculated. As an important result of Ref. [47], the idea of a stable BH remnant as a candidate to conserve information has been failed which means that if we pick up a time-dependent Gaussian distribution of mass/energy, then it will be possible to find a zero remnant mass, albeit in a long-time limit.

One of the serious problems with these remnants is the probability of their detection. Given that the interactions of $\mathrm{BH}$ remnants are purely gravitational, the cross-section is highly small, and a direct observation of these remnants seems impossible. A possible indirect evidence might be related to the cosmic gravitational wave background. In contrast to photons, the gravitons radiated during evaporation would be instantly frozen. Since, the $\mathrm{BH}$ evaporation finishes when it reduces to a remnant, hence, the graviton spectrum should have a cut-off at the Planck mass. In general, such a cut-off is expected to have a redshift on the order of $10^{14} \mathrm{GeV}$. Moreover, we know that the nature of dark matter is hitherto remained an open problem. There exist many dark matter candidates in which most of them are non-baryonic weakly interacting massive particles. A candidate which is not closely connected to particle physics is the relics of primordial BHs [48]. Some specific inflation models naturally induce a great number of such BHs, e.g. the hybrid inflation model can generally produce a required abundance of primordial BH remnants for them to be the main source of the dark matter [49]. 


\section{THE NBBH TEMPERATURE}

While we do not yet have any credible candidate for a full quantum gravity theory, more phenomenological procedures have tried to investigate micro BHs. The recent anticipations imply the conceivable results of LHC experiments, containing the creation of these objects. The possible experimental production of BHs at particle colliders is one of the most significant subfields in extra dimension models. These newly formed miniature BHs first lose their hairs associated with the multipole and the angular momenta, then classically reach the stable Schwarzschild solutions, and finally evaporate via Hawking radiation up to promising Planck-sized remnants. The Hawking temperature is generally subjected to corrections from many sources, particularly, those related to a BH with the mass of the order of the Planck mass. Hence, the study of $\mathrm{TeV}$-scale BHs in the UHECR and particle colliders requires a perfect examination of how temperature corrections affect $\mathrm{BH}$ thermodynamics.

In the following, with the above motivation, we would like to find the temperature corrections of the NB solution. When the NBBH radiates, its Hawking temperature can be calculated to find

$$
\begin{array}{r}
T_{H}=\left.\frac{1}{4 \pi} \frac{d g_{00}}{d r}\right|_{r=r_{H}}=\frac{M}{4 \sqrt{(\pi \theta)^{3}}\left(r_{H}^{2}+g^{2}\right)^{\frac{5}{2}}}\left[4 r_{H} \sqrt{\pi \theta^{3}}\left(\frac{r_{H}^{2}}{2}-g^{2}\right) \mathcal{E}\left(\frac{r_{H}}{2 \sqrt{\theta}}\right)\right. \\
\left.-r_{H}^{2} e^{-\frac{r_{H}^{2}}{4 \theta}}\left(r_{H}^{4}+2 r_{H}^{2} \theta+r_{H}^{2} g^{2}-4 \theta g^{2}\right)\right] .
\end{array}
$$

For large BHs, $r_{H} / \sqrt{\theta} \gg 1$, and $g=0$, the Gauss error function tends to unity and the exponential term is reduced to zero. Thus, one recovers the standard result for the temperature of a Schwarzschild BH, i.e. $T_{H}=M /\left(2 \pi r_{H}^{2}\right)=1 /\left(4 \pi r_{H}\right)$.

At this stage, the numerical result of the Hawking temperature versus the horizon radius is presented in Fig. 3. This figure shows that the temperature peak drops with increasing the parameter $g$. Therefore, we expect that the NBBHs to be colder than the noncommutative Schwarzschild BHs. In addition, the size and the mass of the NBBH remnant at the final stage of the evaporation increase with increasing the magnetic charge. Hence, the remnant of a NBBH may be big compared to the noncommutative Schwarzschild case.

As an important note, if we had chosen the Hayward solution, as another popular example of regular BHs, solely the mass term would have altered, however the general properties would have directed to entirely comparable consequences to those above. Now, let us consider the metric describing the Noncommutative Hayward (NH) BH, in Schwarzschild coordinates, 


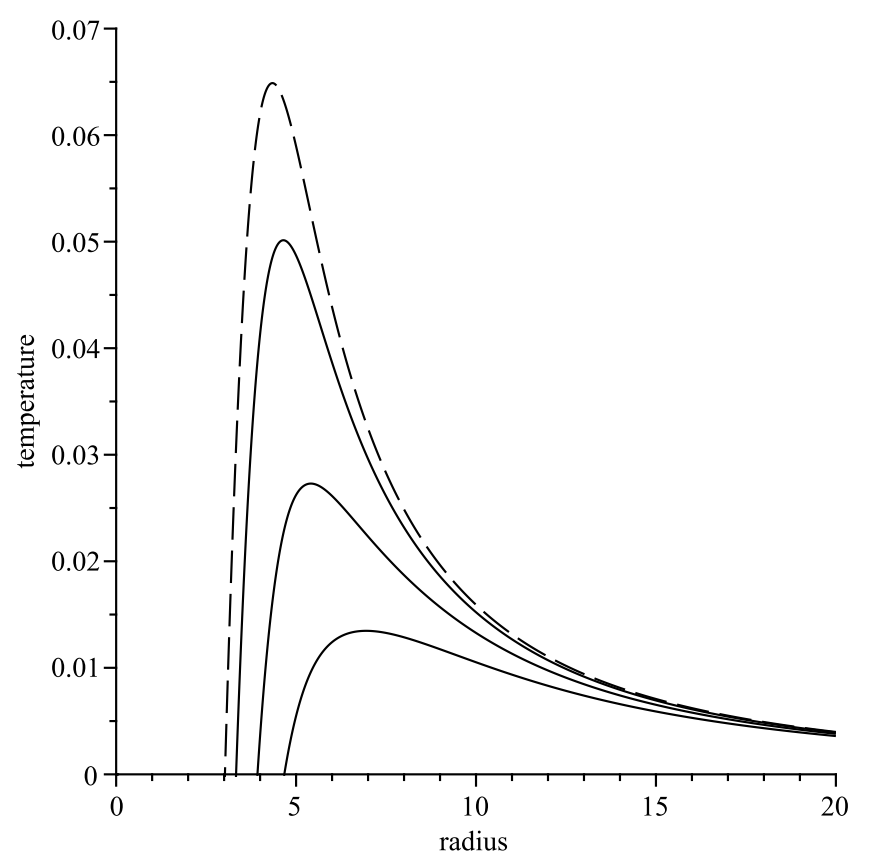

FIG. 3: The temperature $T_{H}$ versus the horizon radius, $r_{H} / \sqrt{\theta}$. We have set $M=10.00 \sqrt{\theta}$. On the right-hand side of the figure, from top to bottom, the solid lines correspond to the NBBH for $g=1.00 \sqrt{\theta}, g=2.00 \sqrt{\theta}$, and $g=3.00 \sqrt{\theta}$, respectively. The dashed line refers to the Schwarzschild case so that it corresponds to $g=0$.

which is immediately given by Eq. (4) with this new mass term

$$
m(r)=M_{\theta}\left(\frac{r^{3}}{r^{3}+g^{\prime 3}}\right)
$$

where $g^{\prime}$ is a positive constant measuring the deviations from the classical Kerr metric. The lack of responsiveness of the results to the kind of the regular $\mathrm{BH}$ can be easily exhibited by plotting the Hawking temperature as a function of radius for NHBHs (see Fig. 4). Comparing these results with the results of Fig. 3 shows the close similarity of outcomes in these two types of regular BHs.

At this point it is worth pointing out that most of the results in the noncommutative framework are confirmed by the so-called Generalized Uncertainty Principle (GUP) context [50 54]. It is widely accepted that the Heisenberg uncertainty principle should be reformulated owing to the noncommutative nature of spacetime at the Planck scale. The application of the GUP to BH thermodynamics has attracted considerable attention in the literature which leads to significant modifications to the emission process, particularly at the final stages of the $\mathrm{BH}$ evaporation (there is a large body of literature on this subject; see for example, [55 58]). As a result of GUP effects on this issue, we have shown that a modification of the de Broglie relation and corresponding commutation relations in the quantum tunneling framework of the $\mathrm{BH}$ evaporation lead to correlations between emitted 


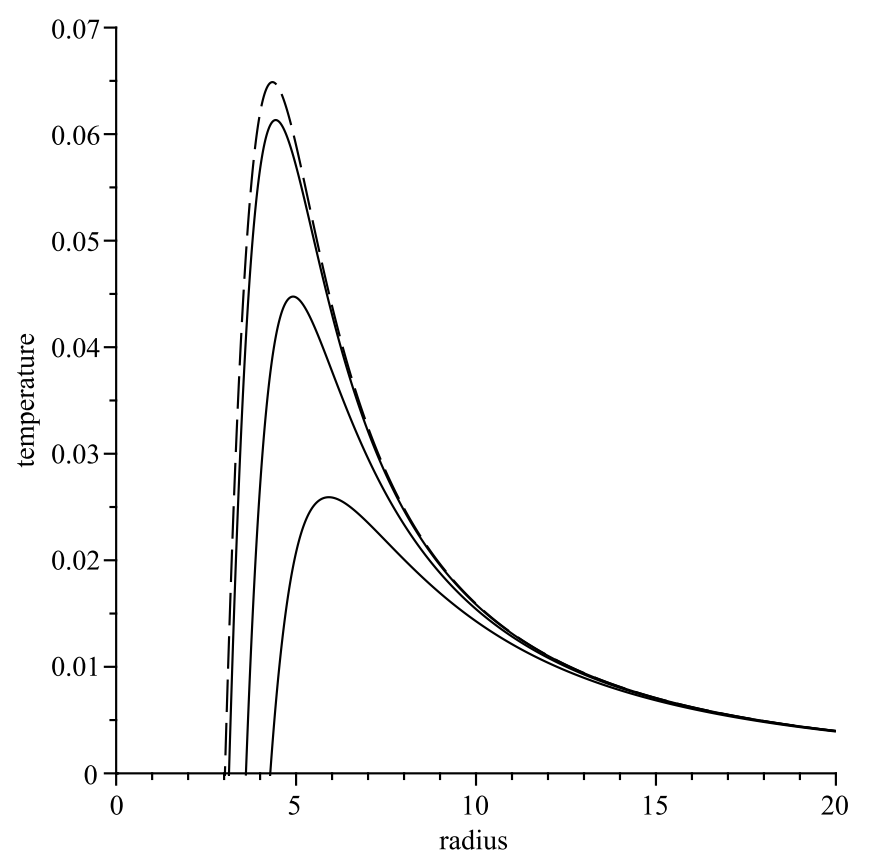

FIG. 4: The temperature $T_{H}$ versus the horizon radius, $r_{H} / \sqrt{\theta}$. We have set $M=10.00 \sqrt{\theta}$. On the right-hand side of the figure, from top to bottom, the solid lines correspond to the NHBH for $g^{\prime}=1.00 \sqrt{\theta}, g^{\prime}=2.00 \sqrt{\theta}$ and $g^{\prime}=3.00 \sqrt{\theta}$, respectively. The dashed line refers to the Schwarzschild case so that it corresponds to $g^{\prime}=0$.

modes of evaporation [59 61]. In this setup, information leaks out of the BH in the form of non-thermal GUP correlations and, on the other hand, the inclusion of quantum gravity effects as the GUP expression can halt the evaporation process, so that a stable BH remnant is left behind, including a part of the BH information content (see also [46]). In addition, recently, the authors of Ref. [62] investigated the GUP effect on the thermodynamics of a Schwarzschild-Tangherlini BH and found that the GUP corrected Hawking temperature is smaller than the original case; it goes to zero when the mass of the $\mathrm{BH}$ reaches a minimal value, which is supported by the results obtained in the framework of the inspired noncommutativity. Also, the results of their study concerning the possibilities to observe a micro $\mathrm{BH}$ in the LHC have shown that the minimum energy for the production of the $\mathrm{BH}$ is larger than the current energy scales of LHC.

As a final remark, our results are supported by the results obtained in the framework of gravity's rainbow [63 65$]$. Ali and his coworkers [63 65$]$ have argued that since a remnant depends critically on the structure of the rainbow functions, therefore a remnant is formed for all black objects in the context of gravity's rainbow and this is a model-independent phenomenon. Their calculations have shown that the behavior of Hawking's radiation changes considerably near the Planck scale in gravity's rainbow such that black objects do not evap- 
orate completely and a remnant is left. Moreover, they have found that the mass of their remnant is greater than the energy scale at which experiments were carried out at the LHC [66].

\section{SUMMARY}

In summary, we have applied the noncommutativity effects to Bardeen BHs. The noncommutative effects become susceptible when the mass of the $\mathrm{BH}$ reaches the order of the Planck scale, it stops radiating and leads to a BH remnant. It is concluded that, for an adequately large magnetic charge of a NBBH there is a linear relationship between the remnant mass and the remnant radius that is just the same as appeared in the relation between the horizon radius and the BH mass for the standard Schwarzschild case. We have found that the temperature peak of the NBBH decreases as the parameter $g$ increases. Thus, a $\mathrm{NBBH}$ is colder than a noncommutative Schwarzschild BH. In this setup, the final stage of the evaporation of a noncommutative regular $\mathrm{BH}$ is a remnant in which it has an increasing size with raising its own characteristic parameter. As a consequence, in the theory of noncommutative regular BHs, the minimum value of energy for the production of such a $\mathrm{BH}$ at the current energy scales of LHC is larger, so the possibility for its detection is less.

\section{Acknowledgments}

Financial support by Lahijan Branch, Islamic Azad University Grant No. 17.20.5.3517 is gratefully acknowledged. The authors thank to C. Bambi for valuable suggestions.

[1] Ansoldi, S. 2008, arXiv:0802.0330

[2] Bardeen, J. M., 1968, in Conference Proceedings of GR5, Tbilisi, USSR, p.174

[3] Ayón-Beato, E., \& García, A. 2000, Phys. Lett. B, 493, 149

[4] Hayward, S. A. 2006, Phys. Rev. Lett. , 96, 031103

[5] Bambi, C., \& Modesto, L. 2013, Phys. Lett. B, 721, 329

[6] Neves, J. C. S., \& Saa, A. 2014, Phys. Lett. B, 734, 44 
[7] Azreg-Aïnou, M. 2014, Phys. Rev. D , 90, 064041

[8] Frolov, V. P. 2014, JHEP, 2014, 49

[9] Amir, M., \& Ghosh, S. G. 2015, arXiv:1503.08553

[10] Lorenzo, T. D., Giusti, A., \& Speziale, S. 2016, Gen. Rel. Grav., 48, 31

[11] Ghosh, S. G., \& Amir, M. 2015, Eur. Phys. J. C, 75, 553

[12] Garcia, A., et al. 2015, J. Math. Phys., 56, 032501

[13] Toshmatov, B., et al. 2014, Phys. Rev. D , 89, 104017

[14] Ghosh, S. G. 2015, Eur. Phys. J. C, 75, 532

[15] Stuchlik, Z., \& Schee, J. 2015, Int. J. Mod. Phys. D, 24, 1550020

[16] Nicolini, P. 2005, J. Phys. A, 38, L631

[17] Nicolini, P., Smailagic, A., \& Spallucci, E. 2006, Phys. Lett. B, 632, 547

[18] Ansoldi, S., et al. 2007, Phys. Lett. B, 645, 261

[19] Nozari, K., \& Mehdipour, S. H. 2008, Class. Quant. Grav., 25, 175015

[20] Nicolini, P. 2009, Int. J. Mod. Phys. A, 24, 1229

[21] Snyder, H. S. 1947, Phys. Rev., 71, 38

[22] DeWitt, B. S. 1964, Phys. Rev. Lett. , 13, 114

[23] Yoneya, T. 1976, Prog. Theor. Phys., 56, 1310

[24] Rovelli, C. 1993, Nucl. Phys. B, 405, 797

[25] Fontanini, M., Spallucci, E., \& Padmanabhan, T. 2006, Phys. Lett. B, 633, 627

[26] Smailagic, A., \& Spallucci, E. 2003, J. Phys. A, 36, L467

[27] Smailagic, A., \& Spallucci, E. 2003, J. Phys. A, 36, L517

[28] Smailagic, A., \& Spallucci, E. 2004, J. Phys. A, 37, 7169

[29] Hawking, S. W. 1975, Comm. Math. Phys., 43, 199

[30] Preskill, J. 1992, arXiv:hep-th/9209058

[31] Page, D. N. 1993, Phys. Rev. Lett. , 71, 3743

[32] Stephens, C. R., 't Hooft, G., \& Whiting, B. F. 1994, Class. Quant. Grav., 11, 621

[33] Russo, J. G. 2005, arXiv:hep-th/0501132

[34] Chen, P., Ong, Y. C., \& Yeom, D.-h. 2015, Phys. Rep., 603, 1

[35] Dymnikova, I., \& Khlopov, M. 2015, Int. J. Mod. Phys. D, 24, 1545002

[36] Banks, T., O'Loughlin, M., \& Strominger, A. 1993, Phys. Rev. D , 47, 4476

[37] Paul, A., \& Majhi, B. R. 2016, arXiv:1601.07310 
[38] Hinchliffe, I., Kersting, N., \& Ma, Y. L. 2004, Int. J. Mod. Phys. A, 19, 179

[39] Rizzo, T. G. 2006, JHEP, 0609, 021

[40] Casadio, R., \& Nicolini, P. 2008, JHEP, 0811, 072

[41] Spallucci, E., Smailagic, A., \& Nicolini, P. 2009, Phys. Lett. B, 670, 449

[42] Antoniadis, I. 1990, Phys. Lett. B, 246, 377

[43] Witten, E. 1996, Nucl. Phys. B, 471, 135

[44] Arkani-Hamed, N., Dimopoulos, S., \& Dvali, G. R. 1998, Phys. Lett. B, 429, 263

[45] Randall, L., \& Sundrum, R. 1999, Phys. Rev. Lett. , 83, 3370

[46] Nozari, k., \& Mehdipour, S. H. 2009, JHEP, 03, 061

[47] Mehdipour, S. H. 2010, Phys. Rev. D , 81, 124049

[48] Zeldovich, Y. B., \& Novikov, I. D. 1966, Sov. Astron., 10, 602

[49] Linde, A. 2000, Phys. Rep., 333, 575

[50] Amati, D., Ciafaloni, M., \& Veneziano, G. 1989, Phys. Lett. B, 216, 41

[51] Konishi, K., Paffuti, G., \& Provero, P. 1990, Phys. Lett. B, 234, 276

[52] Maggiore, M. 1994, Phys. Rev. D , 49, 5182

[53] Kempf, A., Mangano, G., \& Mann, R. B. 1995, Phys. Rev. D , 52, 1108

[54] Scardigli, F. 1999, Phys. Lett. B, 452, 39

[55] Adler, R. J., Chen, P., \& Santiago, D. I. 2001, Gen. Rel. Grav., 33, 2101

[56] Medved, A. J. M., \& Vagenas, E. C. 2004, Phys. Rev. D , 70, 124021

[57] Bolen, B., \& Cavaglia, M. 2005, Gen. Rel. Grav., 37, 1255

[58] Nozari, K., \& Mehdipour, S. H. 2006, Int. J. Mod. Phys. A, 21, 4979

[59] Nozari, K., \& Mehdipour, S. H. 2008, Europhys. Lett., 84, 20008

[60] Mehdipour, S. H. 2009, Int. J. Mod. Phys. A, 24, 5669

[61] Mehdipour, S. H. 2012, Can. J. Phys., 90, 425

[62] Feng, Z. W., et al. 2015, arXiv:1512.09219

[63] Ali, A. F. 2014, Phys. Rev. D , 89, 104040

[64] Ali, A. F., Faizal, M., \& Khalil, M. M. 2014, JHEP, 12, 159

[65] Ali, A. F., Faizal, M., \& Khalil, M. M. 2015, Nucl. Phys. B, 894, 341

[66] Ali, A. F., Faizal, M., \& Khalil, M. M. 2015, Phys. Lett. B, 743, 295

[67] Note that, a micro BH can be produced at the LHC just under the condition of $E_{c m}>M_{0}$, where $E_{c m}$ is the parton-parton center-of-mass energy which is proportional to the TeV energy 
scale. 